\title{
Zero Correlation Linear Cryptanalysis with Reduced Data Complexity
}

\author{
Andrey Bogdanov ${ }^{1, \star}$ and Meiqin Wang ${ }^{1,2, \star}$ \\ ${ }^{1}$ KU Leuven, ESAT/COSIC and IBBT, Belgium \\ 2 Shandong University, Key Laboratory of Cryptologic Technology and Information \\ Security, Ministry of Education, Shandong University, Jinan 250100, China
}

\begin{abstract}
Zero correlation linear cryptanalysis is a novel key recovery technique for block ciphers proposed in 5. It is based on linear approximations with probability of exactly $1 / 2$ (which corresponds to the zero correlation). Some block ciphers turn out to have multiple linear approximations with correlation zero for each key over a considerable number of rounds. Zero correlation linear cryptanalysis is the counterpart of impossible differential cryptanalysis in the domain of linear cryptanalysis, though having many technical distinctions and sometimes resulting in stronger attacks.

In this paper, we propose a statistical technique to significantly reduce the data complexity using the high number of zero correlation linear approximations available. We also identify zero correlation linear approximations for 14 and 15 rounds of TEA and XTEA. Those result in keyrecovery attacks for 21-round TEA and 25-round XTEA, while requiring less data than the full code book. In the single secret key setting, these are structural attacks breaking the highest number of rounds for both ciphers.

The findings of this paper demonstrate that the prohibitive data complexity requirements are not inherent in the zero correlation linear cryptanalysis and can be overcome. Moreover, our results suggest that zero correlation linear cryptanalysis can actually break more rounds than the best known impossible differential cryptanalysis does for relevant block ciphers. This might make a security re-evaluation of some ciphers necessary in the view of the new attack.
\end{abstract}

Keywords: block ciphers, key recovery, linear cryptanalysis, zero correlation linear cryptanalysis, data complexity, TEA, XTEA.

\section{Introduction}

\subsection{Motivation}

Differential and linear cryptanalyses [3,31] are the two basic tools for evaluating the security of block ciphers such as the former U.S. encryption standard DES as well as its successor AES. While DES was developed at the time when differential

\footnotetext{
* Corresponding authors.
} 
and linear cryptanalyses were not publicly known, the design of AES provably addresses these attacks.

Design strategies have been proposed such as the wide-trail design strategy [14] or decorrelation theory [43] to make ciphers resistant to the basic flavours of differential and linear cryptanalysis. However, a proof of resistance according to these strategies does not necessarily imply resistance to the extensions of these techniques such as impossible differential cryptanalysis [1,7] and the recently proposed zero correlation linear cryptanalysis [5].

Standard differential cryptanalysis uses differentials with probabilities significantly higher than those expected for a randomly drawn permutation. Similarly, basic linear cryptanalysis uses linear approximations whose probabilities detectably deviate from $1 / 2$. At the same time, impossible differential cryptanalysis and zero correlation linear cryptanalysis are based on structural deviations of another kind: Differentials with zero probability are targeted in impossible differential cryptanalysis and linear approximations with probability of exactly $1 / 2$ correlation are exploited in zero correlation linear cryptanalysis. Thus, zero correlation linear cryptanalysis can be seen as the counterpart of impossible differential cryptanalysis in the domain of linear cryptanalysis.

The name of the attack originated from the notion of correlation [12]35]: If $\frac{1+c}{2}$ is the probability for a linear approximation to hold, $c$ is called the correlation of this linear approximation. Clearly, putting $c=0$ yields an unbiased linear approximation of probability $1 / 2$, or a zero correlation linear approximation.

Impossible differential cryptanalysis has been known to the cryptographic community since over a decade now. It has turned out a highly useful tool of attacking block ciphers [2, 16, 28, 30, 42. In fact, among meet-in-the-middle [15] and multiset-type attacks [19], it is the impossible differential cryptanalysis [29] that breaks the highest numbers of rounds of AES-128 and AES-256 in the classical single-key attack model as to date, the recent biclique cryptanalysis [4] being the notable exception though.

Zero correlation linear cryptanalysis is a novel promising attack technique that bears some technical similarities to impossible differential cryptanalysis but has its theoretical foundation in a different mathematical theory. Despite its newness, it has already been demonstrated to successfully apply to round-reduced AES and CLEFIA even in its basic form [5, which is highly motivating for further studies.

In this paper, we show how to remove the data requirement of the full codebook which was the major limitation of basic zero correlation linear cryptanalysis [5. As an application of zero correlation linear cryptanalysis and this data complexity reduction technique, we propose attacks against round-reduced TEA and XTEA. For both ciphers, we can cryptanalyze more rounds than it was previously possible using less than the full code book.

\subsection{Contributions}

The work at hand has two major contributions. 
Data complexity reduction for zero correlation linear cryptanalysis. The data requirements of the full codebook have been a crucial limitation for the recent zero correlation linear cryptanalysis to become a major cryptanalytic technique, though the length of the fundamental property (the length of the zero correlation linear approximation) was demonstrated to be comparable to that of impossible differentials for several cipher structures [5]. Overcoming this annoying limitation, a statistical technique of data complexity reduction for zero correlation linear cryptanalysis is the first contribution of this paper.

The data complexity reduction technique is based on the fact that, like any exploitable impossible differential, a typical zero correlation linear approximation is truncated: That is, once a zero correlation linear approximation has been identified that holds for all keys, it will as a rule imply an entire class of similar zero correlation linear approximations to exist. Those can be typically obtained by just changing several bits of the input mask, output mask or both. In other words, in most practical cases, there will be multiple zero correlation linear approximations available to the adversary which has been ignored by the previous analysis.

However, unlike in impossible differential cryptanalysis, the actual value of the correlation has to be estimated in zero correlation linear cryptanalysis and it is not enough to just wait for the impossible event to occur. In fact, the idea we use for zero correlation linear cryptanalysis is more similar to that of multiple linear cryptanalysis: We estimate the correlation of each individual linear approximation using a limited number of texts. Then, for a group of zero correlation linear approximations (i.e. for the right key), we expect the cumulative deviation of those estimations from 0 to be lower than that for a group of randomly chosen linear approximations (i.e. for a wrong key). Given the statistical behaviour of correlation for a randomly drawn permutation [13, 36, this consideration results in a $\chi^{2}$ statistic and allows for a theoretical analysis of the complexity and error probabilities of a zero correlation linear attack that are confirmed by experiments.

\section{Zero correlation linear cryptanalysis of round-reduced TEA and} XTEA. TEA (Tiny Encryption Algorithm) is one of the first lightweight block ciphers. It is a 64-bit block cipher based on a balanced Feistel-type network with a simple ARX round function. TEA has 64 rounds and accepts a key of 128 bits. It favours both efficient hardware [23] and software implementations. TEA was designed by Wheeler and Needham and proposed at FSE'94 [44. It was used in Microsoft's Xbox gaming console for checking software authenticity until its weakness as a hash function was used [4] to compromise the chain of trust. The block cipher XTEA [34 is the fixed version of TEA eliminating this property (having the same number rounds, block size, and key size). TEA and XTEA being rather popular ciphers, both are implemented in the Linux kernel.

Similarly to the complementation property of DES, TEA has an equivalent key property and its effective key size is 126 bits (compared to 128 bits suggested by the nominal key input size) 24]. Kelsey, Scheier and Wagner [25] proposed a practical related-key attack on the full TEA. Using complementation 
Table 1. Summary of cryptanalytic results on round-reduced TEA* and XTEA in the single-key setting

\begin{tabular}{|c|c|c|c|c|c|c|}
\hline attack & \#rounds & data & comp. compl. & memory & Pr[success $]$ & ref. \\
\hline \multicolumn{7}{|c|}{ TEA } \\
\hline impossible differential & 11 & $2^{52.5} \mathrm{CP}$ & $2^{84}$ & $\overline{\mathrm{NA}}$ & $\overline{\mathrm{NA}}$ & 33 \\
\hline truncated differential & 17 & $1920 \mathrm{CP}$ & $2^{123.37}$ & NA & NA & 21 \\
\hline impossible differential & 17 & $2^{57} \mathrm{CP}$ & $2^{106.6}$ & $2^{49}$ & NA & 9 \\
\hline zero correlation linear & 21 & $2^{62.62} \mathrm{KP}$ & $2^{121.52}$ & negligible & 0.846 & this paper \\
\hline zero correlation linear & 23 & $2^{64}$ & $2^{119.64}$ & negligible & 1 & this paper \\
\hline \multicolumn{7}{|c|}{ XTEA } \\
\hline impossible differential & 14 & $2^{62.5} \mathrm{CP}$ & $2^{85}$ & $\overline{\mathrm{NA}}$ & $\mathrm{NA}$ & 33 \\
\hline truncated differential & 23 & $2^{20.55} \mathrm{CP}$ & $2^{120.65}$ & NA & 0.969 & 21 \\
\hline meet-in-the-middle & 23 & $18 \mathrm{KP}$ & $2^{117}$ & & $1-2^{-1025}$ & 38 \\
\hline impossible differential & 23 & $2^{62.3} \mathrm{CP}$ & $2^{114.9}$ & $2^{94.3}$ & NA & 9 \\
\hline impossible differential & 23 & $2^{63}$ & $2^{101} \mathrm{MA}+2^{105.6}$ & $2^{103}$ & NA & 9 \\
\hline zero correlation linear & 25 & $2^{62.62} \mathrm{KP}$ & $2^{124.53}$ & $2^{30}$ & 0.846 & this paper \\
\hline zero correlation linear & 27 & $2^{64}$ & $2^{120.71}$ & negligible & 1 & this paper \\
\hline
\end{tabular}

cryptanalysis 8, up to 36 rounds of XTEA can be attacked with related keys for all keys. The work [8] also contains related-key attacks for up to 50 rounds of XTEA working for a weak key class.

In the classical single-key setting, however, by far not all rounds of TEA are broken by structural attacks (whereas the effective key size is 126 bits for the full cipher). The truncated differential result on 17 rounds remains the best cryptanalysis of TEA [21]. Impossible differential cryptanalysis [9] has yielded a faster attack against 17 rounds of TEA. Similarly, 23 rounds of XTEA have been cryptanalyzed so far using truncated differential [21, impossible differential [9] and well as meet-in-the-middle attacks [38. That is, for both TEA and XTEA, there has been no progress in terms of the number of attacked rounds since 2003.

In this paper, using zero correlation linear cryptanalysis, we cryptanalyze 21 rounds of TEA and 25 rounds of XTEA with $2^{62.62}$ known plaintexts (in contrast to chosen texts required in impossible differential cryptanalysis). Certainly, zero correlation linear cryptanalysis for lower number of rounds yields a lower data complexity for both TEA and XTEA. Moreover, unlike most impossible differential attacks including those on TEA and XTEA 9, zero correlation linear cryptanalysis is able to profit from the full code available. If all $2^{64}$ texts are available to the adversary, we propose zero correlation linear cryptanalysis for 23 rounds of TEA and 27 rounds of XTEA. Our cryptanalytic results are summarized and compared to previous cryptanalysis in Table 1.

As opposed to the initial intuition expressed in [5], both major contributions of this work - the data complexity reduction and the new attacks on more rounds of TEA and XTEA — demonstrate that zero correlation linear cryptanalysis can actually perform better than impossible differential cryptanalysis. Moreover, we expect the security of more ciphers to be reevaluated under the consideration of zero correlation linear cryptanalysis. 


\subsection{Outline}

We start with a review of the basic zero correlation linear cryptanalysis for block ciphers in Section 2. In Section 3, we introduce a $\chi^{2}$ statistical technique for reducing the data requirements of zero correlation linear cryptanalysis and thoroughly investigate its complexity. In Section 4, the 14- and 15-round zero correlation linear approximations are demonstrated for block ciphers TEA and XTEA. Section 5 gives several zero correlation key recoveries for round-reduced TEA and XTEA. The full version [6] of this paper is available online and contains proofs of some technical statements as well as further zero correlation linear attacks on round-reduced TEA and XTEA.

\section{Basic Zero Correlation Linear Cryptanalysis}

Zero correlation linear cryptanalysis has been introduced in [5]. Below we briefly review its basic ideas and methods.

\subsection{Linear Approximations with Correlation Zero}

Consider an $n$-bit block cipher $f_{K}$ with key $K$. Let $P$ denote a plaintext which is mapped to ciphertext $C$ under key $K, C=f_{K}(P)$. If $\Gamma_{P}$ and $\Gamma_{C}$ are nonzero plaintext and ciphertext linear masks of $n$ bit each, we denote by $\Gamma_{P} \rightarrow \Gamma_{C}$ the linear approximation

$$
\Gamma_{P}^{T} P \oplus \Gamma_{C}^{T} C=0 .
$$

Here, $\Gamma_{A}^{T} A$ denotes the multiplication of the transposed bit vector $\Gamma_{A}$ (linear mask for $A$ ) by a column bit vector $A$ over $\mathbb{F}_{2}$. The linear approximation $\Gamma_{P} \rightarrow$ $\Gamma_{C}$ has probability

$$
p_{\Gamma_{P}, \Gamma_{C}}=\operatorname{Pr}_{P \in \mathbb{F}_{2}^{n}}\left\{\Gamma_{P}^{T} P \oplus \Gamma_{C}^{T} C=0\right\}
$$

The value

$$
c_{\Gamma_{P}, \Gamma_{C}}=2 p_{\Gamma_{P}, \Gamma_{C}}-1
$$

is called the correlation (or bias) of linear approximation $\Gamma_{P} \rightarrow \Gamma_{C}$. Note that $p_{\Gamma_{P}, \Gamma_{C}}=1 / 2$ is equivalent to zero correlation $c_{\Gamma_{P}, \Gamma_{C}}=0$ :

$$
p_{\Gamma_{P}, \Gamma_{C}}=\operatorname{Pr}_{P \in \mathbb{F}_{2}^{n}}\left\{\Gamma_{P}^{T} P \oplus \Gamma_{C}^{T} C=0\right\}=1 / 2 .
$$

In fact, for a randomly drawn permutation of sufficiently large bit size $n$, zero is the most frequent single value of correlation for a nontrivial linear approximation. Correlation goes to small values for increasing $n$, the probability to get exactly zero decreases as a function of $n$ though. More precisely, the probability of the linear approximation $\Gamma_{P} \rightarrow \Gamma_{C}$ with $\Gamma_{P}, \Gamma_{C} \neq 0$ to have zero correlation has been shown [5. Proposition 2] to be approximated by

$$
\frac{1}{\sqrt{2 \pi}} 2^{\frac{4-n}{2}} .
$$




\subsection{Two Examples}

Given a randomly chosen permutation, however, it is hard to tell a priori which of its nontrivial linear approximations in particular has zero correlation. At the same time, it is often possible to identify groups of zero correlation linear approximations for a block cipher $f_{K}$ once it has compact description with a distinct structure. Moreover, in many interesting cases, these linear approximations will have zero correlation for any key $K$. Here are two examples provided in [5]:

- AES: The data transform of AES has a set of zero correlation linear approximations over 4 rounds ( 3 full rounds appended by 1 incomplete rounds with MixColumns omitted). If $\Gamma$ and $\Gamma^{\prime}$ are 4-byte column linear masks with exactly one nonzero byte, then each of the linear approximations $(\Gamma, 0,0,0) \rightarrow$ $\left(\Gamma^{\prime}, 0,0,0\right)$ over 4 AES rounds has zero correlation [5, Theorem 2].

- CLEFIA-type GFNs: CLEFIA-type generalized Feistel networks [40] (also known as type-2 GFNs with 4 lines [45) have zero correlation linear approximations over 9 rounds, if the underlying F-functions of the Feistel construction are invertible. For $a \neq 0$, the linear approximations $(a, 0,0,0) \rightarrow$ $(0,0,0, a)$ and $(0,0, a, 0) \rightarrow(0, a, 0,0)$ over 9 rounds have zero correlation [5. Theorem 1].

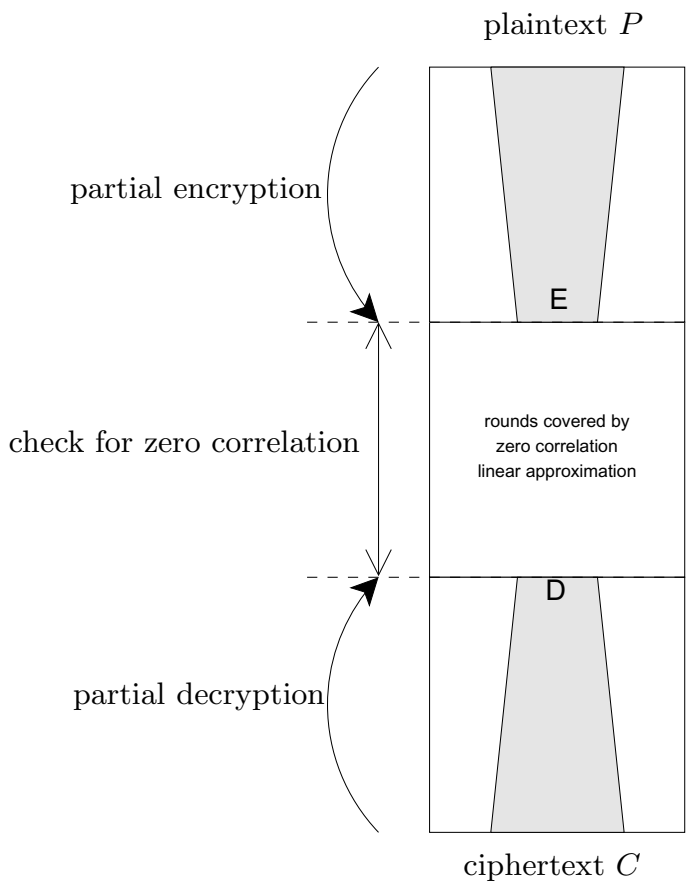

Fig. 1. High-level view of key recovery in zero correlation linear cryptanalysis 


\subsection{Key Recovery with Zero Correlation Linear Approximations}

Based on linear approximations of correlation zero, a technique similar to Matsui's Algorithm 2 [31] can be used for key recovery. Let the adversary have $N$ known plaintext-ciphertexts and $\ell$ zero correlation linear approximations $\left\{\Gamma_{E} \rightarrow \Gamma_{D}\right\}$ for a part of the cipher, with $\ell=\left|\left\{\Gamma_{E} \rightarrow \Gamma_{D}\right\}\right|$. The linear approximations $\left\{\Gamma_{E} \rightarrow \Gamma_{D}\right\}$ are placed in the middle of the attacked cipher. Let $E$ and $D$ be the partial intermediate states of the data transform at the boundaries of the linear approximations.

Then the key can be recovered using the following approach (see also Figure@):

1. Guess the bits of the key needed to compute $E$ and $D$. For each guess:

(a) Partially encrypt the plaintexts and partially decrypt the ciphertexts up to the boundaries of the zero correlation linear approximation $\Gamma_{E} \rightarrow \Gamma_{D}$.

(b) Estimate the correlations $\left\{\hat{c}_{\Gamma_{E}, \Gamma_{D}}\right\}$ of all linear approximations in $\left\{\Gamma_{E} \rightarrow\right.$ $\left.\Gamma_{D}\right\}$ for the key guess using the partially encrypted and decrypted values $E$ and $D$ by counting how many times $\Gamma_{E}^{T} E \oplus \Gamma_{D}^{T} D$ is zero over $N$ input/output pairs, see (11) and (2).

(c) Perform a test on the estimated correlations $\left\{\hat{c}_{\Gamma_{E}, \Gamma_{D}}\right\}$ for $\left\{\Gamma_{E} \rightarrow \Gamma_{D}\right\}$ to tell of the estimated values of $\left\{\hat{c}_{\Gamma_{E}, \Gamma_{D}}\right\}$ are compatible with the hypothesis that all of the actual values of $\left\{c_{\Gamma_{E}, \Gamma_{D}}\right\}$ are zero.

2. Test the surviving key candidates against a necessary number of plaintextciphertext pairs according to the unicity distance for the attacked cipher.

Step 1(c) of the technique above relies on an efficient test distinguishing between the hypothesis that $\left\{c_{\Gamma_{E}, \Gamma_{D}}\right\}$ are all zero and the alternative hypothesis. The work [5] requires the exact evaluation of the correlation value (defined by the probability of a linear approximation) and the data complexity is restricted to $N=2^{n}$ in [5]. Thus, a small number $\ell$ of linear approximations is usually enough in [5] and $\hat{c}_{\Gamma_{E}, \Gamma_{D}}=c_{\Gamma_{E}, \Gamma_{D}}$, though the data complexity of the full codebook is too restrictive.

For most ciphers (including the examples of Subsection 2.2), however, a large number $\ell$ of zero correlation linear approximations is available. This freedom is not used in [5]. At the same time, it has been shown in the experimental work [10] that any value of correlation can be used for key recovery in a linear attack with reduced data complexity, once enough linear approximations are available. Despite its convincing experimental evidence, [10] gives no theoretical data complexity estimations and does not provide any ways of constructing linear approximations with certain properties.

In the next section of this paper, we provide a framework for reducing the data complexity $N$ if many zero correlation linear approximations are known.

\section{Reduction of Data Complexity with Many Approximations}

\subsection{Distinguishing between Two Normal Distributions}

Consider two normal distributions: $\mathcal{N}\left(\mu_{0}, \sigma_{0}\right)$ with mean $\mu_{0}$ and standard deviation $\sigma_{0}$, and $\mathcal{N}\left(\mu_{1}, \sigma_{1}\right)$ with mean $\mu_{1}$ and standard deviation $\sigma_{1}$. A sample $s$ is 
drawn from either $\mathcal{N}\left(\mu_{0}, \sigma_{0}\right)$ or $\mathcal{N}\left(\mu_{1}, \sigma_{1}\right)$. It has to be decided if this sample is from $\mathcal{N}\left(\mu_{0}, \sigma_{0}\right)$ or from $\mathcal{N}\left(\mu_{1}, \sigma_{1}\right)$. The test is performed by comparing the value $s$ to some threshold value $t$. Without loss of generality, assume that $\mu_{0}<\mu_{1}$. If $s \leq t$, the test returns " $s \in \mathcal{N}\left(\mu_{0}, \sigma_{0}\right)$ ". Otherwise, if $s>t$, the test returns $" s \in \mathcal{N}\left(\mu_{1}, \sigma_{1}\right)$ ". There will be error probabilities of two types:

$$
\begin{aligned}
& \beta_{0}=\operatorname{Pr}\left\{" s \in \mathcal{N}\left(\mu_{1}, \sigma_{1}\right) " \mid s \in \mathcal{N}\left(\mu_{0}, \sigma_{0}\right)\right\}, \\
& \beta_{1}=\operatorname{Pr}\left\{" s \in \mathcal{N}\left(\mu_{0}, \sigma_{0}\right) " \mid s \in \mathcal{N}\left(\mu_{1}, \sigma_{1}\right)\right\} .
\end{aligned}
$$

Here a condition is given on $\mu_{0}, \mu_{1}, \sigma_{0}$, and $\sigma_{1}$ such that the error probabilities are $\beta_{0}$ and $\beta_{1}$. The proof immediately follows from the basics of probability theory (see e.g. [18, 20, ) and is given in the full version [6 of the paper for completeness.

Proposition 1. For the test to have error probabilities of at most $\beta_{0}$ and $\beta_{1}$, the parameters of the normal distributions $\mathcal{N}\left(\mu_{0}, \sigma_{0}\right)$ and $\mathcal{N}\left(\mu_{1}, \sigma_{1}\right)$ with $\mu_{0} \neq \mu_{1}$ have to be such that

$$
\frac{z_{1-\beta_{1}} \sigma_{1}+z_{1-\beta_{0}} \sigma_{0}}{\left|\mu_{1}-\mu_{0}\right|}=1
$$

where $z_{1-\beta_{1}}$ and $z_{1-\beta_{0}}$ are the quantiles of the standard normal distribution.

\subsection{A Known Plaintext Distinguisher with Many Zero Correlation Linear Approximations}

Let the adversary be given $N$ known plaintext-ciphertext pairs and $\ell$ zero correlation linear approximations for an $n$-bit block cipher. The adversary aims to distinguish between this cipher and a randomly drawn permutation.

The procedure is as follows. For each of the $\ell$ given linear approximations, the adversary computes the number $T_{i}$ of times the linear approximations are fulfilled on $N$ plaintexts, $i \in\{1, \ldots, \ell\}$. Each $T_{i}$ suggests an empirical correlation value $\hat{c}_{i}=2 \frac{T_{i}}{N}-1$. Then, the adversary evaluates the statistic:

$$
\sum_{i=1}^{\ell} \hat{c}_{i}^{2}=\sum_{i=1}^{\ell}\left(2 \frac{T_{i}}{N}-1\right)^{2} .
$$

It is expected that for the cipher with $\ell$ known zero correlation linear approximations, the value of statistic (5) will be lower than that for $\ell$ linear approximations of a randomly drawn permutation. In a key-recovery setting, the right key will result in statistic (5) being among the lowest values for all candidate keys if $\ell$ is high enough. In the sequel, we treat this more formally.

\subsection{Correlation under Right and Wrong Keys}

Consider the key recovery procedure outlined in Subsection 2.3 given $N$ known plaintext-ciphertext pairs. There will be two cases: 
- Right key guess: Each of the values $\hat{c}_{i}$ in (5) approximately follows the normal distribution with zero mean and standard deviation $1 / \sqrt{N}$ with good precision (c.f. e.g. [22, 39]) for sufficiently large $N$ :

$$
\hat{c}_{i} \sim \mathcal{N}(0,1 / \sqrt{N})
$$

- Wrong key guess: Each of the values $\hat{c}_{i}$ in (5) approximately follows the normal distribution with mean $c_{i}$ and standard deviation $1 / \sqrt{N}$ for sufficiently large $N$ :

$$
\hat{c}_{i} \sim \mathcal{N}\left(c_{i}, 1 / \sqrt{N}\right) \text { with } c_{i} \sim \mathcal{N}\left(0,2^{-n / 2}\right),
$$

where $c_{i}$ is the exact value of the correlation which is itself distributed as $\mathcal{N}\left(0,2^{-n / 2}\right)$ over random permutations with $n \geq 5-$ a result due to [13, 36. Thus, our wrong key hypothesis is that for each wrong key, the adversary obtains a permutation with linear properties close to those of a randomly chosen permutation.

\subsection{Distribution of the Statistic}

Based on these distributions of $\hat{c}_{i}$, we now derive the distributions of statistic (5) in these two cases.

Right Key Guess. In this case, we deal with $\ell$ zero correlation linear approximations:

$$
\sum_{i=1}^{\ell} \hat{c}_{i}^{2} \sim \sum_{i=1}^{\ell} \mathcal{N}^{2}(0,1 / \sqrt{N})=\frac{1}{N} \sum_{i=1}^{\ell} \mathcal{N}^{2}(0,1)=\frac{1}{N} \chi_{\ell}^{2}
$$

where $\chi_{\ell}^{2}$ is the $\chi^{2}$-distribution with $\ell$ degrees of freedom which has mean $\ell$ and standard deviation $\sqrt{2 \ell}$, assuming the independency of underlying distributions. For sufficiently large $\ell, \chi_{\ell}^{2}$ converges to the normal distribution. That is, $\chi_{\ell}^{2}$ approximately follows $\mathcal{N}(\ell, \sqrt{2 \ell})$, and:

$$
\sum_{i=1}^{\ell} \hat{c}_{i}^{2} \sim \frac{1}{N} \chi_{\ell}^{2} \approx \frac{1}{N} \mathcal{N}(\ell, \sqrt{2 \ell})=\mathcal{N}\left(\frac{\ell}{N}, \frac{\sqrt{2 \ell}}{N}\right) .
$$

Proposition 2. Consider $\ell$ nontrivial zero correlation linear approximations for a block cipher with a fixed key. If $N$ is the number of known plaintext-ciphertext pairs, $T_{i}$ is the number of times such a linear approximation is fulfilled for $i \in$ $\{1, \ldots, \ell\}$, and $\ell$ is high enough, then, assuming the counters $T_{i}$ are independent, the following approximate distribution holds for sufficiently large $N$ and $n$ :

$$
\sum_{i=1}^{\ell}\left(2 \frac{T_{i}}{N}-1\right)^{2} \sim \mathcal{N}\left(\frac{\ell}{N}, \frac{\sqrt{2 \ell}}{N}\right)
$$


Wrong Key Guess. The wrong key hypothesis is that we deal with pick a permutation at random for each wrong key. Therefore, the $\ell$ given linear approximations will have randomly drawn correlations, under this hypothesis. Thus, as mentioned above:

$$
\sum_{i=1}^{\ell} \hat{c}_{i}^{2} \sim \sum_{i=1}^{\ell} \mathcal{N}^{2}\left(c_{i}, 1 / \sqrt{N}\right), \text { where } c_{i} \sim \mathcal{N}\left(0,2^{-n / 2}\right) .
$$

First, we show that the underlying distribution of $\hat{c}_{i}$ is actually normal with mean 0 . Then we show that the sum approximately follows $\chi^{2}$-distribution assuming the independency of underlying distributions, and can be approximated by another normal distribution.

Since

$$
\begin{aligned}
\mathcal{N}\left(c_{i}, 1 / \sqrt{N}\right) & =c_{i}+\mathcal{N}(0,1 / \sqrt{N}) \\
& =\mathcal{N}\left(0,1 / \sqrt{2^{n}}\right)+\mathcal{N}(0,1 / \sqrt{N}) \\
& =\mathcal{N}\left(0, \sqrt{1 / N+1 / 2^{n}}\right)
\end{aligned}
$$

the distribution above is a $\chi^{2}$-distribution with $\ell$ degrees of freedom up to a factor, under the independency assumption:

$$
\begin{aligned}
\sum_{i=1}^{\ell} \mathcal{N}^{2}\left(c_{i}, 1 / \sqrt{N}\right) & =\sum_{i=1}^{\ell} \mathcal{N}^{2}\left(0, \sqrt{\frac{1}{N}+\frac{1}{2^{n}}}\right) \\
& =\left(\frac{1}{N}+\frac{1}{2^{n}}\right) \sum_{i=1}^{\ell} \mathcal{N}^{2}(0,1) \\
& =\left(\frac{1}{N}+\frac{1}{2^{n}}\right) \chi_{\ell}^{2} .
\end{aligned}
$$

As for the right keys, for sufficiently large $\ell, \chi_{\ell}^{2}$ can be approximated by the normal distribution with mean $\ell$ and standard deviation $\sqrt{2 \ell}$. Thus:

$$
\begin{aligned}
\sum_{i=1}^{\ell} \hat{c}_{i}^{2} & \sim\left(\frac{1}{N}+\frac{1}{2^{n}}\right) \chi_{\ell}^{2} \approx\left(\frac{1}{N}+\frac{1}{2^{n}}\right) \mathcal{N}(\ell, \sqrt{2 \ell}) \\
& =\mathcal{N}\left(\frac{\ell}{N}+\frac{\ell}{2^{n}}, \frac{\sqrt{2 \ell}}{N}+\frac{\sqrt{2 \ell}}{2^{n}}\right) .
\end{aligned}
$$

Proposition 3. Consider $\ell$ nontrivial linear approximations for a randomly drawn permutation. If $N$ is the number of known plaintext-ciphertext pairs, $T_{i}$ is the number of times a linear approximation is fulfilled for $i \in\{1, \ldots, \ell\}$, and $\ell$ is high enough, then, assuming the independency of $T_{i}$, the following approximate distribution holds for sufficiently large $N$ and $n$ :

$$
\sum_{i=1}^{\ell}\left(2 \frac{T_{i}}{N}-1\right)^{2} \sim \mathcal{N}\left(\frac{\ell}{N}+\frac{\ell}{2^{n}}, \frac{\sqrt{2 \ell}}{N}+\frac{\sqrt{2 \ell}}{2^{n}}\right) .
$$

\subsection{Data Complexity of the Distinguisher}

Combining Propositions 2 and 3 with Proposition 1, one obtains the condition:

$$
\frac{z_{1-\beta_{1}}\left(\frac{\sqrt{2 \ell}}{N}+\frac{\sqrt{2 \ell}}{2^{n}}\right)+z_{1-\beta_{0}} \frac{\sqrt{2 \ell}}{N}}{\left(\frac{\ell}{N}+\frac{\ell}{2^{n}}\right)-\frac{\ell}{N}}=1 .
$$


The left part of this equation can be simplified to

$$
\frac{2^{n+0.5}}{N \sqrt{\ell}}\left(z_{1-\beta_{0}}+z_{1-\beta_{1}}\right)+\frac{z_{1-\beta_{1}} \sqrt{2}}{\sqrt{\ell}}
$$

which yields

Theorem 1. With the assumptions of Propositions 1 to 3, using $\ell$ nontrivial zero correlation linear approximations, to distinguish between a wrong key and a right key with probability $\beta_{1}$ of false positives and probability $\beta_{0}$ of false negatives, a number $N$ of known plaintext-ciphertext pairs is sufficient if the following condition is fulfilled:

$$
\frac{2^{n+0.5}}{N \sqrt{\ell}}\left(z_{1-\beta_{0}}+z_{1-\beta_{1}}\right)+\frac{z_{1-\beta_{1}} \sqrt{2}}{\sqrt{\ell}}=1 .
$$

The success probability of an attack is defined by the probability $\beta_{0}$ of false negatives. The probability $\beta_{1}$ of false positives determines the number of surviving key candidates and, thus, influences the computational complexity of the key recovery.

\section{Linear Approximations with Correlation Zero for TEA and XTEA}

In [5], a sufficient condition is given for a linear approximation to have a correlation of zero. Namely, if for a linear approximation there exist no linear characteristics with non-zero correlation contributions, then the correlation of the linear approximation is exactly zero.

\subsection{The Block Ciphers TEA and XTEA}

TEA is a 64-round iterated block cipher with 64-bit block size and 128-bit key which consist of four 32-bit words $K[0], K[1], K[2]$ and $K[3]$. TEA does not have any iterative key schedule algorithm. Instead, the key words are used directly in round functions. The round constant is derived from the constant $\delta=9 e 3779 b 9_{x}$ and the round number. We denote the input and the output of the $r$-th round for $1 \leq r \leq 64$ by $\left(L_{r}, R_{r}\right)$ and $\left(L_{r+1}, R_{r+1}\right)$, respectively. $L_{r+1}=R_{r}$ and $R_{r+1}$ is computed as follows:

$$
R_{r+1}= \begin{cases}L_{r}+\left(\left(\left(R_{r} \ll 4\right)+K[0]\right) \oplus\left(R_{r}+i \cdot \delta\right) \oplus\left(R_{r} \gg 5+K[1]\right)\right) & r=2 i-1, \\ L_{r}+\left(\left(\left(R_{r} \ll 4\right)+K[2]\right) \oplus\left(R_{r}+i \cdot \delta\right) \oplus\left(R_{r} \gg 5+K[3]\right)\right) & r=2 i, 1 \leq i \leq 32 .\end{cases}
$$

Like TEA, XTEA is also a 64-round Feistel cipher with 64-bit block and 128-bit key. Its 128 -bit secret key $K$ is represented by four 32-bit words $K[0], K[1], K[2]$ and $K[3]$ as well. The derivation of the subkey word number is slightly more complex though. The input of the $r$-th round is $\left(L_{r}, R_{r}\right)$ and the output is $\left(L_{r+1}, R_{r+1}\right)$. Again, $L_{r+1}=R_{r}$ and $R_{r+1}$ is derived as:

$R_{r+1}=\left\{\begin{array}{l}L_{r}+\left(\left(\left(R_{r} \ll 4 \oplus R_{r} \gg 5\right)+R_{r}\right) \oplus((i-1) \cdot \delta+K[((i-1) \cdot \delta \ll 11) \& 3])\right) \quad r=2 i-1, \\ L_{r}+\left(\left(\left(R_{r} \ll 4 \oplus R_{r} \gg 5\right)+R_{r}\right) \oplus(i \cdot \delta+K[(i \cdot \delta \ll 11) \& 3])\right) \quad r=2 i, 1 \leq i \leq 32 .\end{array}\right.$

These round functions of TEA and XTEA are illustrated in Figure 2. 

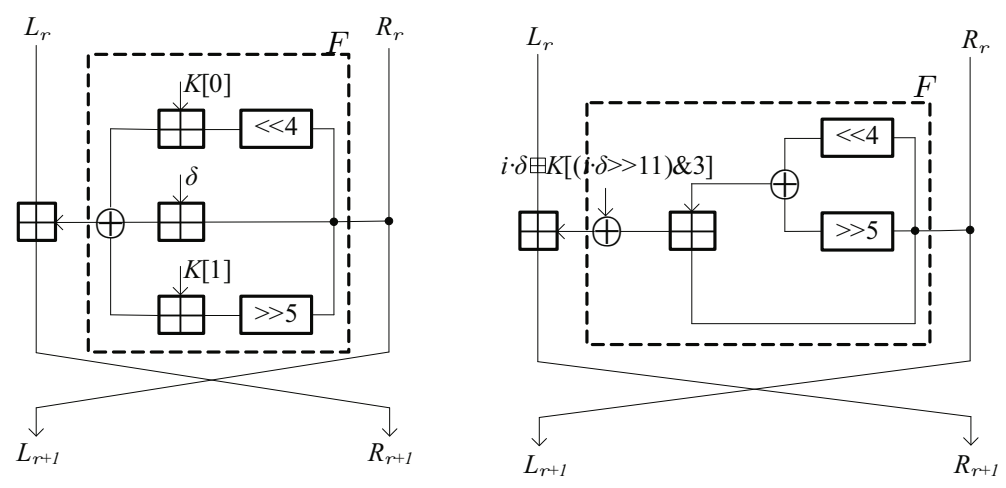

Fig. 2. Round function for TEA(left) and XTEA(right)

\subsection{Notations}

To demonstrate zero correlation linear approximations for TEA and XTEA, we will need the following notations (the least significant bit of a word has number $0)$ :

- $e_{i, \sim}$ is a 32-bit word that has zeros in bits $(i+1)$ to 31 , one in bit $i$ and undefined values in bits 0 to $(i-1)$,

- $e_{i \sim j}$ is a 32 -bit word that has zeros in bits $(i+1)$ to 31 and bits 0 to $(j-1)$, a one in bit $i$ and undefined values in bits $j$ to $(i-1)$ for $j<i$,

$-\bar{e}_{i, \sim}$ is a 32 -bit word that has zeros in bits $(i+1)$ to 31 , undefined values in bits 0 to $i$,

$-?$ is an undefined value,

$-X^{i \sim j}$ is bits from $j$ to $i$ of the value $X, j<i$, and

$-X^{i}$ is the value of bit $i$ of $X$.

\subsection{Linear Approximation of Modular Addition}

Here, we first demonstrate the properties of linear approximations with non-zero correlation over the modular addition, which is the only nonlinear part of the TEA and XTEA transformation (summarized as Property 1). Then we use it to show a condition for linear approximation with non-zero correlation for one round of TEA and XTEA (stated as Property 2).

For the modular addition of two $n$-bit inputs $x$ and $y$, the output $z$ can be computed as:

$$
z=(x+y) \bmod 2^{n} .
$$

We denote the mask values for $x, y$ and $z$ as $\Gamma x, \Gamma y$ and $\Gamma z$, respectively $\left(x, y, z, \Gamma x, \Gamma y\right.$, and $\left.\Gamma z \in \mathbb{F}_{2}^{n}\right)$. The linear approximation for the modular addition is then $\Gamma x^{T} \cdot x \oplus \Gamma y^{T} \cdot y=\Gamma z^{T} \cdot z$ and is referred to as

$$
+:(\Gamma x \mid \Gamma y) \rightarrow \Gamma z .
$$


Property 1 (Modular addition). In any linear approximation $(\Gamma x \mid \Gamma y) \rightarrow \Gamma z$ of the modular addition with a non-zero correlation, the most significant non-zero mask bit for $\Gamma x, \Gamma y$ and $\Gamma z$ is the same.

Property 1 is proven in the full version [6] of the paper.

\subsection{Linear Approximation of One TEA/XTEA Round}

Using Property 1 for modular addition, as all other operations in TEA and XTEA are linear, we can derive conditions on a special class of approximations with non-zero correlation for the round function of TEA and XTEA. See Figures 4 and 3 for an illustration.

As in Subsection 4.1, the input and output of round $r$ in TEA and XTEA are $\left(L_{r} \mid R_{r}\right)$ and $\left(L_{r+1} \mid R_{r+1}\right)$, respectively. Correspondingly, $\left(\Gamma_{r}^{L} \mid \Gamma_{r}^{R}\right)$ and $\left(\Gamma_{r+1}^{L} \mid \Gamma_{r+1}^{R}\right)$ are input and output linear masks of the round. So the linear approximation over the round is

$$
\text { (X)TEA round } r:\left(\Gamma_{r}^{L} \mid \Gamma_{r}^{R}\right) \rightarrow\left(\Gamma_{r+1}^{L} \mid \Gamma_{r+1}^{R}\right)
$$

and has the following

Property 2 (One round). If $\Gamma_{r}^{L}=e_{i, \sim}$ and $\Gamma_{r}^{R}=e_{j, \sim},(j<i)$, then one needs $\Gamma_{r+1}^{R}=e_{i, \sim}$ and $\Gamma_{r+1}^{L}=e_{i, \sim} \oplus e_{i+5 \sim 5}$ for the approximation to have a nonzero correlation. Similarly, for the decryption round function of TEA, if the input mask and the output mask for round $r$ are $\left(\Gamma_{r}^{L} \mid \Gamma_{r}^{R}\right)$ and $\left(\Gamma_{r+1}^{L} \mid \Gamma_{r+1}^{R}\right)$, respectively. If $\Gamma_{r}^{R}=e_{i, \sim}$ and $\Gamma_{r}^{L}=e_{j, \sim},(j<i)$, then we have $\Gamma_{r+1}^{L}=e_{i, \sim}$ and $\Gamma_{r+1}^{R}=e_{i, \sim} \oplus e_{i+5 \sim 5}$.

\subsection{Zero Correlation Approximations for 14 and 15 Rounds of TEA/XTEA}

With the one-round property of linear approximation in TEA and XTEA derived in the previous subsection, we can identify zero correlation approximations over 14 and 15 rounds of both TEA and XTEA.

Proposition 4. Over 15 rounds of TEA and XTEA, any linear approximation with input mask $\left(\Gamma_{1}^{R} \mid \Gamma_{1}^{L}\right)=(1 \mid 0)$ and output mask $\left(\Gamma_{15}^{R} \mid \Gamma_{15}^{L}\right)=\left(0 \mid e_{1, \sim}\right)$ has a correlation of exactly zero. Moreover, over 14 rounds of TEA and XTEA, any linear approximation with input mask $\left(\Gamma_{1}^{R} \mid \Gamma_{1}^{L}\right)=(1 \mid 0)$ and output mask $\left(\Gamma_{14}^{R} \mid \Gamma_{14}^{L}\right)=\left(e_{1, \sim} \mid \bar{e}_{5, \sim}\right)$ has zero correlation.

Proof. First, we follow the linear approximation in the forward direction. From $\Gamma_{1}^{L}=0$ and $\Gamma_{1}^{R}=1$, it is obtained that $\Gamma_{2}^{L}=0$ and $\Gamma_{2}^{R}=1$, then we get $\Gamma_{3}^{L}=1 \oplus(1<<5)$ and $\Gamma_{3}^{R}=1$. From Property 2 , $\Gamma_{3}^{L}=1 \oplus(1<<5)$ and $\Gamma_{3}^{R}=1$, then we have $\Gamma_{4}^{R}=e_{5, \sim}$ and $\Gamma_{4}^{L}=e_{5, \sim} \oplus e_{5+5 \sim 5} \oplus 1=e_{10, \sim}$. Similarly, we get $\left(\Gamma_{5}^{R} \mid \Gamma_{5}^{L}\right)=\left(e_{10, \sim} \mid e_{15, \sim}\right),\left(\Gamma_{6}^{R} \mid \Gamma_{6}^{L}\right)=\left(e_{15, \sim} \mid e_{20, \sim}\right),\left(\Gamma_{7}^{R} \mid \Gamma_{7}^{L}\right)=\left(e_{20, \sim} \mid e_{25, \sim}\right)$, $\left(\Gamma_{8}^{R} \mid \Gamma_{8}^{L}\right)=\left(e_{25, \sim} \mid e_{30, \sim}\right)$ and $\left(\Gamma_{9}^{R} \mid \Gamma_{9}^{L}\right)=\left(e_{30, \sim} \mid ?\right)$. 


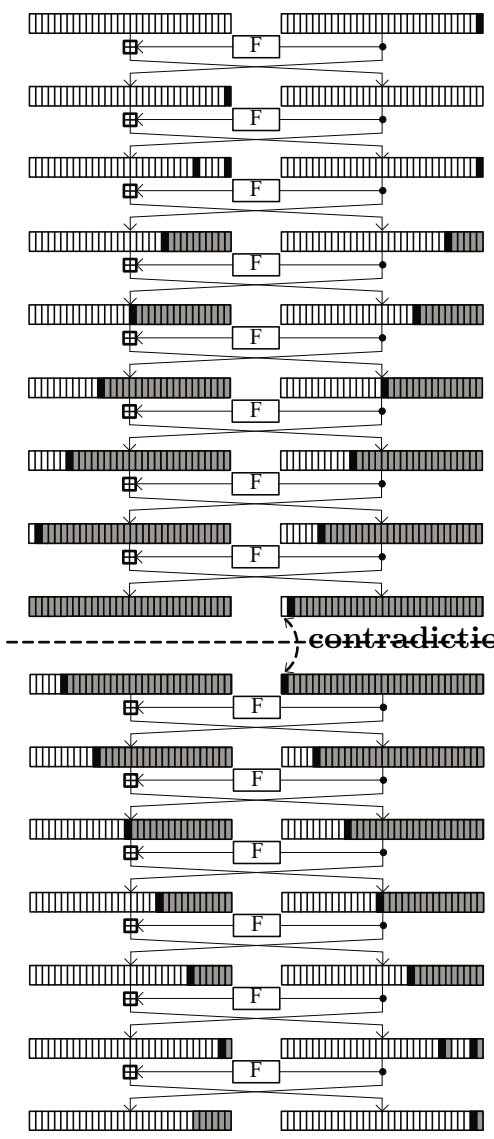

Fig. 3. Zero correlation linear approximation for 14-round TEA and XTEA (grey undefined bits, black - bits set to 1 )

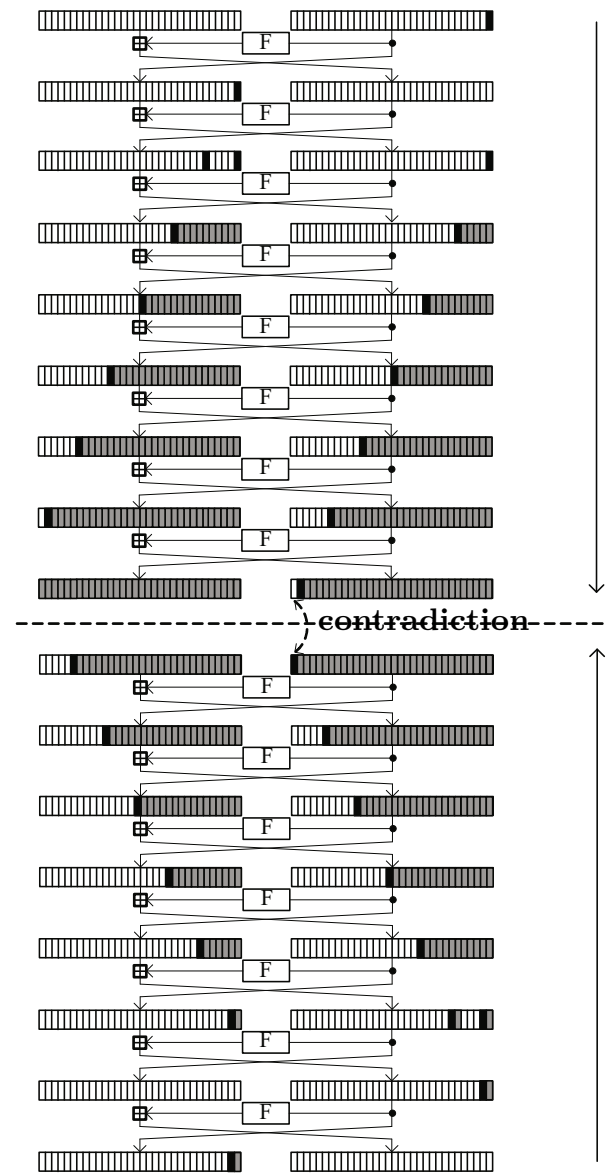

Fig. 4. Zero correlation linear approximation for 15-round TEA and XTEA (grey undefined bits, black - bits set to 1 )

Second, we follow the 7-round linear approximation in the backward direction. From $\Gamma_{16}^{L}=e_{1, \sim}$ and $\Gamma_{16}^{R}=0$, we can derive that $\left(\Gamma_{15}^{R} \mid \Gamma_{15}^{L}\right)=\left(e_{1, \sim} \mid 0\right)$, $\left(\Gamma_{14}^{R} \mid \Gamma_{14}^{L}\right)=\left(e_{1, \sim} \oplus e_{6 \sim 5} \mid e_{1, \sim}\right),\left(\Gamma_{13}^{R} \mid \Gamma_{13}^{L}\right)=\left(e_{11, \sim} \mid e_{6, \sim}\right),\left(\Gamma_{12}^{R} \mid \Gamma_{12}^{L}\right)=\left(e_{16, \sim} \mid e_{11, \sim}\right)$, $\left(\Gamma_{11}^{R} \mid \Gamma_{11}^{L}\right)=\left(e_{21, \sim} \mid e_{16, \sim}\right),\left(\Gamma_{10}^{R} \mid \Gamma_{10}^{L}\right)=\left(e_{26, \sim} \mid e_{21, \sim}\right)$ and $\left(\Gamma_{9}^{R} \mid \Gamma_{9}^{L}\right)=\left(e_{31, \sim} \mid e_{26, \sim}\right)$.

From the forward direction, the most significant bit of $\Gamma_{9}^{R}$ has to be zero, and from the backward direction, the most significant bit of $\Gamma_{9}^{R}$ has to be one. This yields a contradiction and shows that there are no characteristics for this linear approximation. By the sufficient condition of [5] for constructing zero correlation linear approximations, this is enough for the approximation to have correlation zero. So the linear approximation for 15-round TEA and XTEA with the input mask $(1 \mid 0)$ and the output mask $\left(0 \mid e_{1, \sim}\right)$ has zero correlation. By restricting this linear approximation to 14 rounds and adding several undefined bits to the output mask, one gets all the claims of the proposition. 
There are only 2 zero correlation linear approximations of this form over 15 rounds. We note however that there are $2^{7}$ different zero correlation linear approximations over 14 rounds of both TEA and XTEA. They can be generated by setting the undefined bits (depicted in gray in Figure 3 and Figure 4) to different values.

\section{Zero Correlation Linear Cryptanalysis of Round-Reduced (X)TEA}

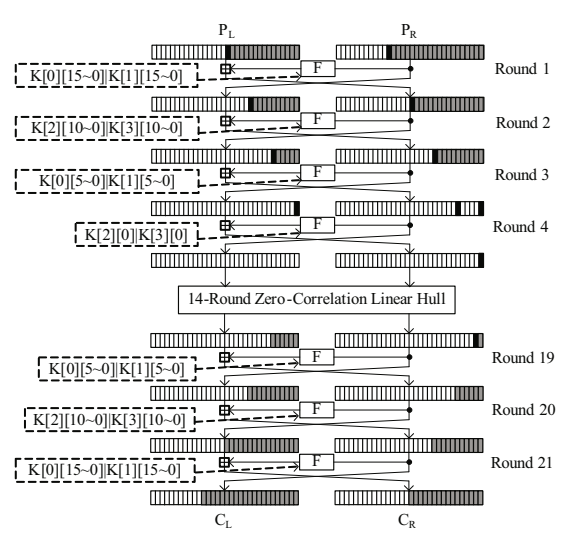

Fig. 5. Key recovery for 21 rounds of TEA. For the estimation of correlation, grey and black bits need to be computed and white bits are irrelevant. Uses the zero correlation approximation of Figure 3 .

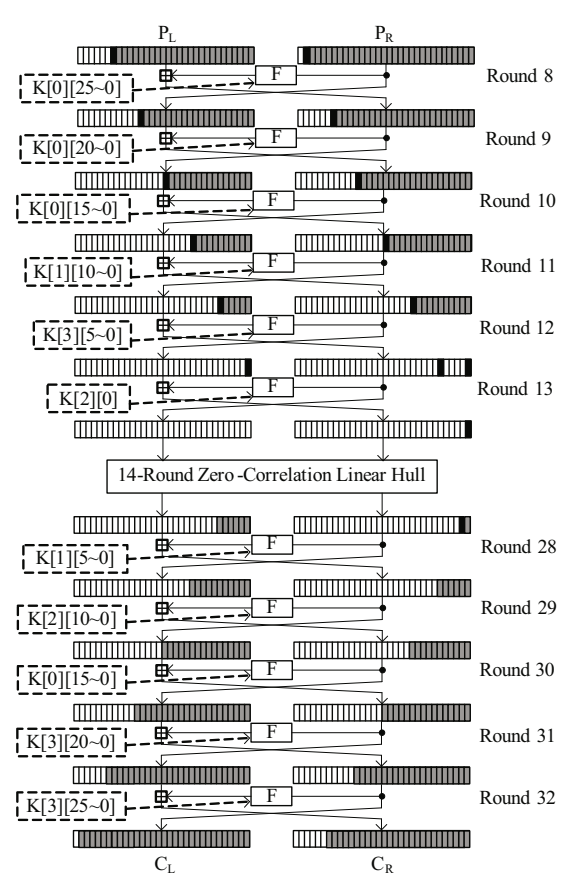

Fig. 6. Key recovery for 25 rounds of XTEA For the estimation of correlation, grey and black bits need to be computed and white bits are irrelevant. Uses the zero correlation approximation of Figure 3

\subsection{Key Recovery for 21 Rounds of TEA}

For the cryptanalysis of 21-round TEA, we use the 14-round zero correlation approximations of the type depicted in Figure 3 of Subsection 4.5. The availability of many such approximations allows us to use the data complexity reduction technique of Section 3 .

We place the 14-round zero correlation linear approximations in the middle of the 21 -round TEA. It covers rounds 5 to 18 . Following the procedure outlined in 
Subsection 2.3, up to the boundaries of the linear approximations, we partially encrypt over the 4 first rounds 1 to 4 and partially decrypt over the 3 last rounds 19 to 21 . The attack is illustrated in Figure 5 .

The linear approximations involve 9 state bits: $R_{5}^{0}, R_{19}^{1 \sim 0}$, and $L_{19}^{5 \sim 0}$. In the corresponding 9 bits of the input and output masks, only 7 can take on 0 and 1 values: $\Gamma_{19}^{R^{0}}$ and $\Gamma_{19}^{L^{5} 0}$. For the evaluation of the linear approximations from a plaintext-ciphertext pair, we guess 54 key bits $K_{0}^{15 \sim 0}, K_{1}^{15 \sim 0}, K_{2}^{10 \sim 0}$, and $K_{3}^{10 \sim 0}$. The attack flow is as follows given $N$ known plaintext-ciphertext pairs.

For each possible guess of the 54 -bit subkey $\kappa=\left(K_{0}^{15 \sim 0}\left|K_{1}^{15 \sim 0}\right| K_{2}^{10 \sim 0} \mid K_{3}^{10 \sim 0}\right)$ :

1. Allocate a 128-bit counter $W$ and set it to zero. $W$ will contain the $\chi^{2}$ statistic for the subkey guess $\kappa$.

2. Allocate a 64 -bit counter $V[x]$ for each of $2^{9}$ possible values of

$$
x=\left(R_{5}^{0}\left|R_{19}^{1 \sim 0}\right| L_{19}^{5 \sim 0}\right)
$$

and set it to $0 . V[x]$ will contain the number of times the partial state value $x$ occurs for $N$ texts.

3. For each of $N$ plaintext-ciphertext pairs: partially encrypt 4 rounds and partially decrypt 3 rounds, obtain the 9-bit value for $x=\left(R_{5}^{0}\left|R_{19}^{1} \sim^{0}\right| L_{19}^{5 \sim 0}\right)$ and add one to the counter $V[x]$.

4. For each of $2^{7}$ zero correlation linear approximations:

(a) Set the 64-bit counter $U$ to zero.

(b) For $2^{9}$ values of $x$, verify if the linear approximation holds. If so, add $V[x]$ to $U$.

(c) $W=W+(2 \cdot U / N-1)^{2}$.

5. If $W<t$, then $\kappa$ is a possible subkey candidate and all cipher keys it is compatible with are tested exhaustively against a maximum of 3 plaintextciphertext pairs.

The correct 54-bit subkey $\kappa$ is likely to be among the candidates with the $\chi^{2}$ statistic $W$ lower than the threshold $t=\sigma_{0} \cdot z_{1-\beta_{0}}+\mu_{0}=\frac{\sqrt{2 l}}{N} \cdot z_{1-\beta_{0}}+\frac{l}{N}=$ $\frac{\sqrt{2 \cdot 2^{7}}}{N} \cdot z_{1-\beta_{0}}+\frac{2^{7}}{N}$, see Subsection 3.1 with its Proposition 1 as well as Theorem 1 In this attack, we set $\beta_{0}=2^{-2.7}, \beta_{1}=2^{-4.49}$ and get $z_{1-\beta_{0}}=1, z_{1-\beta_{1}}=1.7$. Note once again that $n=64$ and $\ell=2^{7}$. Theorem 1 suggests the data complexity of $N=2^{62.62}$ known plaintext-ciphertexts with those parameters. The decision threshold is $t=2^{-55.56}$.

The computational complexity is dominated by Steps 3 and 5 . The computational complexity $T_{3}$ of Step 3 is $2^{54}$ times 7 half-round encryptions for each of $N$ texts. This gives $T_{3}=2^{54} \cdot 2^{62.62} \cdot 7 \cdot 0.5 / 21=2^{114.03} 21$-round TEA encryptions.

One in $1 / \beta_{1}=2^{4.49}$ keys is expected to survive the test against zero correlation. The remaining key space is be covered by exhaustive search which is performed in Step 5. The computational complexity $T_{5}$ of Step 5 is about $T_{5}=2^{126-4.49}=2^{121.51} 21$-round encryptions using the equivalent key property. $T_{5}$ dominates the total computational complexity. 
Summarizing the attack, its computational complexity is about $2^{121.51}$, data complexity is about $2^{62.62}$ known plaintext-ciphertext pairs, and the memory complexity is negligible. The success probability is about 0.846 .

\subsection{Key Recovery for 25-Round XTEA}

Similarly to the attack on 21 rounds of TEA provided in the previous subsection, we use the 14-round zero correlation linear approximation depicted in Figure 3 to attack 25-round XTEA. Note that the attack covers rounds 8 to 32 . It is illustrated in Figure 6. The linear approximations are placed in rounds 14 to 27. We partially encrypt 6 rounds ( 8 to 13 ) and partially decrypt 5 rounds (28 to 32 ) to evaluate the parity of approximations.

The linear approximations involve 9 bits and in the corresponding 9 bits of the input and output masks, again only 7 can take on 0 and 1 values: $\Gamma_{28}^{R^{0}}$ and $\Gamma_{28}^{L^{5 \sim 0}}$. For the evaluation of the linear approximations from a plaintextciphertext pair, we guess altogether 74 key bits $K_{0}^{25 \sim 0}, K_{1}^{10 \sim 0}, K_{2}^{10 \sim 0}$, and $K_{3}^{25 \sim 0}$. The attack itself is similar to that on 21-round TEA.

For each possible 63 -bit value of $\left(K_{0}^{25 \sim 0}\left|K_{1}^{10 \sim 0}\right| K_{3}^{25 \sim 0}\right)$ :

1. Allocate and set to zero the 32 -bit counter $V_{1}[x]$ for each of $2^{30}$ possible values of

$$
x=\left(R_{13}^{0}\left|R_{13}^{5}\right| L_{13}^{0}\left|R_{30}^{10 \sim 0}\right| L_{30}^{15 \sim 0}\right) .
$$

2. For each of $N$ plaintext-ciphertext pairs: partially encrypt 5 rounds and partially decrypt 3 rounds, obtain 30 -bit $x=\left(R_{13}^{0}\left|R_{13}^{5}\right| L_{13}^{0}\left|R_{30}^{10 \sim 0}\right| L_{30}^{15 \sim 0}\right)$, and add one to $V_{1}[x]$.

3. For each possible 11 bits value of $K_{2}^{10 \sim 0}$ :

(a) Allocate and set to zero a 128-bit counter $W$.

(b) Allocate and set to zero a 64-bit counter $V_{2}[y]$ for each of $2^{9}$ possible values of

$$
y=\left(R_{14}^{0}\left|L_{28}^{5 \sim 0}\right| R_{28}^{1 \sim 0}\right) .
$$

(c) Encrypt one round and decrypt two rounds for $2^{30}$ values for $x$ to get 9 bits of $y$ and add $V_{1}[x]$ to $V_{2}[y]$.

(d) For each of $2^{7}$ zero correlation linear approximations:

i. Set the 64-bit counter $U$ to zero.

ii. For $2^{9}$ values of $y$, verify if the linear approximation holds. If so, add $V_{2}[y]$ to the counter $U$.

iii. $W=W+(2 \cdot U / N-1)^{2}$.

(e) If $W<t$, then $\kappa$ is a possible subkey candidate and all cipher keys it is compatible with are tested exhaustively against a maximum of 3 plaintext-ciphertext pairs.

The correct 74-bit subkey is likely to be among the candidates with the $\chi^{2}$ statistic $W$ lower than the threshold $t$. As we again set $\beta_{0}=2^{-2.7}$ and $\beta_{1}=$ $2^{-4.49}$, we obtain $N=2^{62.62}$ and $t=2^{-55.56}$.

The computational complexity is dominated by Step 2 and checking for false positives in Step 3(e). $T_{2}$ of Step 2 is constituted by $2^{63} N$ computations of 5 rounds 
of 25-round XTEA and by $2^{63} N$ increments in the memory of $2^{30} 32$-bit counters. Assuming that one increment of a memory cell costs one XTEA round, we obtain $T_{2}=2^{63} \cdot 2^{62.62} \cdot(5 / 25+1 / 25)=2^{123.56}$. In Step 3(e), the remaining $T_{3(e)}=$ $2^{128-4.49}=2^{123.51}$ keys can checked exhaustively by the same number of 25 -round XTEA encryptions. Thus, the overall computational complexity is about $T_{2}+$ $T_{3(e)}=2^{123.56}+2^{123.51}=2^{124.53} 25$-round XTEA encryptions. The memory complexity is $2^{30} 32$-bit words. Again, the data complexity is about $2^{62.62}$ known plaintext-ciphertext pairs, and the success probability is about 0.846 .

\subsection{Attacking More Rounds with the Full Codebook}

The attacks in the previous subsections use 14-round zero correlation linear approximations to enable data complexity reduction. As we only identified $215-$ round approximations, we cannot use this longer property to attack more rounds and still get a non-negligible decrease in the number of texts required. By taking advantage of the full codebook, we are however able to perform key recovery for up to 23 rounds of TEA and up to 27 rounds of XTEA, see the full version [6] of this paper.

Acknowledgements. We would like to thank Vincent Rijmen and Gregor Leander for insightful discussions. Andrey Bogdanov is postdoctoral fellow of the Fund for Scientific Research - Flanders (FWO). This work has been supported in part by the IAP Programme P6/26 BCRYPT of the Belgian State, by the European Commission under contract number ICT-2007-216676 ECRYPT NoE phase II, by KU Leuven-BOF (OT/08/027), by the Research Council KU Leuven (GOA TENSE), by NSFC Projects (No.61133013, No.61070244 and No.60931160442) as well as Outstanding Young Scientists Foundation Grant of Shandong Province (No.BS2009DX030).

\section{References}

1. Biham, E., Biryukov, A., Shamir, A.: Cryptanalysis of Skipjack Reduced to 31 Rounds Using Impossible Differentials. In: Stern, J. (ed.) EUROCRYPT 1999. LNCS, vol. 1592, pp. 12-23. Springer, Heidelberg (1999)

2. Biham, E., Dunkelman, O., Keller, N.: Related-Key Impossible Differential Attacks on 8-Round AES-192. In: Pointcheval, D. (ed.) CT-RSA 2006. LNCS, vol. 3860, pp. 21-33. Springer, Heidelberg (2006)

3. Biham, E., Shamir, A.: Differential Cryptanalysis of DES-like Cryptosystems. In: Menezes, A., Vanstone, S.A. (eds.) CRYPTO 1990. LNCS, vol. 537, pp. 2-21. Springer, Heidelberg (1991)

4. Bogdanov, A., Khovratovich, D., Rechberger, C.: Biclique Cryptanalysis of the Full AES. In: Lee, D.H. (ed.) ASIACRYPT 2011. LNCS, vol. 7073, pp. 344-371. Springer, Heidelberg (2011)

5. Bogdanov, A., Rijmen, V.: Zero Correlation Linear Cryptanalysis of Block Ciphers. IACR Eprint Archive Report 2011/123 (March 2011)

6. Bogdanov, A., Wang, M.: Zero Correlation Linear Cryptanalysis with Reduced Data Complexity. IACR Eprint Archive Report (2012)

7. Borst, J., Knudsen, L.R., Rijmen, V.: Two Attacks on Reduced IDEA. In: Fumy, W. (ed.) EUROCRYPT 1997. LNCS, vol. 1233, pp. 1-13. Springer, Heidelberg (1997) 
8. Bouillaguet, C., Dunkelman, O., Leurent, G., Fouque, P.-A.: Another Look at Complementation Properties. In: Hong, S., Iwata, T. (eds.) FSE 2010. LNCS, vol. 6147, pp. 347-364. Springer, Heidelberg (2010)

9. Chen, J., Wang, M., Preneel, B.: Impossible Differential Cryptanalysis of Lightweight Block Ciphers TEA, XTEA and HIGHT. IACR Eprint Archive Report 2011/616 (2011)

10. Collard, B., Standaert, F.-X.: Experimenting Linear Cryptanalysis. In: Junod, P., Canteaut, A. (eds.) Advanced Linear Cryptanalysis of Block and Stream Ciphers. Cryptology and Information Security Series, vol. 7. IOS Press (2011)

11. Collard, B., Standaert, F.-X., Quisquater, J.-J.: Improving the Time Complexity of Matsui's Linear Cryptanalysis. In: Nam, K.-H., Rhee, G. (eds.) ICISC 2007. LNCS, vol. 4817, pp. 77-88. Springer, Heidelberg (2007)

12. Daemen, J., Govaerts, R., Vandewalle, J.: Correlation Matrices. In: Preneel, B. (ed.) FSE 1994. LNCS, vol. 1008, pp. 275-285. Springer, Heidelberg (1995)

13. Daemen, J., Rijmen, V.: Probability distributions of correlations and differentials in block ciphers. Journal on Mathematical Cryptology 1(3), 221-242 (2007)

14. Daemen, J., Rijmen, V.: The Design of Rijndael: AES - The Advanced Encryption Standard. Springer (2002)

15. Demirci, H., Selçuk, A.A.: A Meet-in-the-Middle Attack on 8-Round AES. In: Nyberg, K. (ed.) FSE 2008. LNCS, vol. 5086, pp. 116-126. Springer, Heidelberg (2008)

16. Dunkelman, O., Keller, N.: An Improved Impossible Differential Attack on MISTY1. In: Pieprzyk, J. (ed.) ASIACRYPT 2008. LNCS, vol. 5350, pp. 441-454. Springer, Heidelberg (2008)

17. Etrog, J., Robshaw, M.J.B.: On Unbiased Linear Approximations. In: Steinfeld, R., Hawkes, P. (eds.) ACISP 2010. LNCS, vol. 6168, pp. 74-86. Springer, Heidelberg (2010)

18. Feller, W.: An Introduction to Probability Theory and Its Applications, vol. 1. Wiley \& Sons (1968)

19. Dunkelman, O., Keller, N., Shamir, A.: Improved Single-Key Attacks on 8-Round AES-192 and AES-256. In: Abe, M. (ed.) ASIACRYPT 2010. LNCS, vol. 6477, pp. 158-176. Springer, Heidelberg (2010)

20. Hoel, P., Port, S., Stone, C.: Introduction to Probability Theory. Brooks Cole (1972)

21. Hong, S., Hong, D., Ko, Y., Chang, D., Lee, W., Lee, S.: Differential Cryptanalysis of TEA and XTEA. In: Lim, J.-I., Lee, D.-H. (eds.) ICISC 2003. LNCS, vol. 2971, pp. 402-417. Springer, Heidelberg (2004)

22. Junod, P.: On the Complexity of Matsui's Attack. In: Vaudenay, S., Youssef, A.M. (eds.) SAC 2001. LNCS, vol. 2259, pp. 199-211. Springer, Heidelberg (2001)

23. Kaps, J.-P.: Chai-Tea, Cryptographic Hardware Implementations of xTEA. In: Chowdhury, D.R., Rijmen, V., Das, A. (eds.) INDOCRYPT 2008. LNCS, vol. 5365, pp. 363-375. Springer, Heidelberg (2008)

24. Kelsey, J., Schneier, B., Wagner, D.: Key-Schedule Cryptanalysis of IDEA, G-DES, GOST, SAFER, and Triple-DES. In: Koblitz, N. (ed.) CRYPTO 1996. LNCS, vol. 1109, pp. 237-251. Springer, Heidelberg (1996)

25. Kelsey, J., Schneier, B., Wagner, D.: Related-key Cryptanalysis of 3-WAY, BihamDES,CAST, DES-X, NewDES, RC2, and TEA. In: Han, Y., Quing, S. (eds.) ICICS 1997. LNCS, vol. 1334, pp. 233-246. Springer, Heidelberg (1997)

26. Lee, E., Hong, D., Chang, D., Hong, S., Lim, J.: A Weak Key Class of XTEA for a Related-Key Rectangle Attack. In: Nguyên, P.Q. (ed.) VIETCRYPT 2006. LNCS, vol. 4341, pp. 286-297. Springer, Heidelberg (2006) 
27. Lu, J.: Related-key rectangle attack on 36 rounds of the XTEA block cipher. International Journal of Information Security 8(1), 1-11 (2009)

28. Lu, J., Kim, J.-S., Keller, N., Dunkelman, O.: Improving the Efficiency of Impossible Differential Cryptanalysis of Reduced Camellia and MISTY1. In: Malkin, T. (ed.) CT-RSA 2008. LNCS, vol. 4964, pp. 370-386. Springer, Heidelberg (2008)

29. Lu, J., Dunkelman, O., Keller, N., Kim, J.: New Impossible Differential Attacks on AES. In: Chowdhury, D.R., Rijmen, V., Das, A. (eds.) INDOCRYPT 2008. LNCS, vol. 5365, pp. 279-293. Springer, Heidelberg (2008)

30. Mala, H., Dakhilalian, M., Rijmen, V., Modarres-Hashemi, M.: Improved Impossible Differential Cryptanalysis of 7-Round AES-128. In: Gong, G., Gupta, K.C. (eds.) INDOCRYPT 2010. LNCS, vol. 6498, pp. 282-291. Springer, Heidelberg (2010)

31. Matsui, M.: Linear Cryptanalysis Method for DES Cipher. In: Helleseth, T. (ed.) EUROCRYPT 1993. LNCS, vol. 765, pp. 386-397. Springer, Heidelberg (1994)

32. Matsui, M.: The First Experimental Cryptanalysis of the Data Encryption Standard. In: Desmedt, Y.G. (ed.) CRYPTO 1994. LNCS, vol. 839, pp. 1-11. Springer, Heidelberg (1994)

33. Moon, D., Hwang, K., Lee, W., Lee, S., Lim, J.: Impossible Differential Cryptanalysis of Reduced Round XTEA and TEA. In: Daemen, J., Rijmen, V. (eds.) FSE 2002. LNCS, vol. 2365, pp. 49-60. Springer, Heidelberg (2002)

34. Needham, R.M., Wheeler, D.J.: Tea extensions. Technical report, Computer Laboratory, University of Cambridge (October 1997), http://www.cix.co.uk/ klockstone/xtea.pdf

35. Nyberg, K.: Correlation theorems in cryptanalysis. Discrete Applied Mathematics 111(1-2), 177-188 (2001)

36. O'Connor, L.: Properties of Linear Approximation Tables. In: Preneel, B. (ed.) FSE 1994. LNCS, vol. 1008, pp. 131-136. Springer, Heidelberg (1995)

37. Röck, A., Nyberg, K.: Exploiting Linear Hull in Matsui's Algorithm 1. In: WCC 2011 (2011)

38. Sekar, G., Mouha, N., Velichkov, V., Preneel, B.: Meet-in-the-Middle Attacks on Reduced-Round XTEA. In: Kiayias, A. (ed.) CT-RSA 2011. LNCS, vol. 6558, pp. 250-267. Springer, Heidelberg (2011)

39. Selçuk, A.A.: On Probability of Success in Linear and Differential Cryptanalysis. Journal of Cryptology 21(1), 131-147 (2008)

40. Shirai, T., Shibutani, K., Akishita, T., Moriai, S., Iwata, T.: The 128-Bit Blockcipher CLEFIA (Extended Abstract). In: Biryukov, A. (ed.) FSE 2007. LNCS, vol. 4593, pp. 181-195. Springer, Heidelberg (2007)

41. Steil, M.: 17 Mistakes Microsoft Made in the Xbox Security System. Chaos Communication Congress (2005), http://events.ccc.de/congress/2005/fahrplan/events/559.en.html

42. Tsunoo, Y., Tsujihara, E., Shigeri, M., Saito, T., Suzaki, T., Kubo, H.: Impossible Differential Cryptanalysis of CLEFIA. In: Nyberg, K. (ed.) FSE 2008. LNCS, vol. 5086, pp. 398-411. Springer, Heidelberg (2008)

43. Vaudenay, S.: Decorrelation: A Theory for Block Cipher Security. J. Cryptology 16(4), 249-286 (2003)

44. Wheeler, D.J., Needham, R.M.: TEA, a Tiny Encryption Algorithm. In: Preneel, B. (ed.) FSE 1994. LNCS, vol. 1008, pp. 363-366. Springer, Heidelberg (1995)

45. Zheng, Y., Matsumoto, T., Imai, H.: On the Construction of Block Ciphers Provably Secure and Not Relying on Any Unproved Hypotheses. In: Brassard, G. (ed.) CRYPTO 1989. LNCS, vol. 435, pp. 461-480. Springer, Heidelberg (1990) 